\title{
General practice perspectives on a bowel cancer screening quality improvement intervention using the Consolidated Framework for Implementation Research
}

\section{Carol A Holdenª, Deborah Turnbullb, Oliver R Frank ${ }^{c, d}$ and lan Olver ${ }^{b}$}

a South Australian Health and Medical Research Institute, Adelaide

b School of Psychology, University of Adelaide, South Australia

c Discipline of General Practice, Adelaide Medical School, University of Adelaide, South Australia

d Oakden Medical Centre, Adelaide, South Australia

e Corresponding author: carol.holden@sahmri.com

\section{Article history}

Publication date: December 2020 Citation: Holden CA, Turnbull D, Frank OR, Olver I. General practice perspectives on a bowel cancer screening quality improvement intervention using the Consolidated Framework for Implementation Research. Public Health Res Pract. 2020; Online early publication. https://doi. org/10.17061/phrp30452016

\section{Key points}

- Little progress has been made in achieving sustained improvements in colorectal cancer (CRC) screening participation in general practice

- Using the Consolidated Framework for Implementation Research, we identified contextual factors that will allow flexible implementation of a quality improvement intervention

- Adaptations to outer-setting constructs (i.e. beyond the practice setting) may support the implementation of a primary care $\mathrm{CRC}$ quality improvement intervention within the existing organised CRC screening program

\section{Abstract}

Introduction: An understanding of contextual factors that influence whether general practitioners advise their patients to be screened for colorectal cancer (CRC) might guide interventions to increase screening participation from its persistently low rate. We report on the use of a theory-based tool to explore contextual factors that might influence implementation of a novel quality improvement (QI) intervention to increase CRC screening in general practice (CRC-QI). The objective was to identify and incorporate strategies into the intervention that will enable flexible implementation across different practice settings.

Study type: A qualitative study to explore contextual facilitators of, and barriers to, the implementation of a novel CRC-QI intervention.

Methods: Eighteen staff, from three self-nominated general practices, participated in focus group discussions. The Consolidated Framework for Implementation Research (CFIR), which included constructs relevant to CRC screening in primary care, guided the formative evaluation. Findings were aligned to the CFIR model using a deductive thematic analysis.

Results: Contextual facilitators of, and barriers to, the implementation of the CRC-QI intervention were identified in each CFIR domain and CRCrelevant construct. Five consistent themes were identified that potentially influence elements of the CRC-QI intervention: priority setting and incentives, information technology, patient-level barriers, clinical practice, and the National Bowel Cancer Screening Program (NBCSP). Participants proposed that incentive payments and NBCSP policy changes (outer-setting strategies) would facilitate organisational change (inner-setting strategies) and the effective implementation of the CRC-QI intervention. 
Conclusion: There may be an opportunity to better engage general practice in CRC screening via outer-setting constructs that support existing clinical practice. For example, improvements to the National Cancer Screening Register and Quality Improvement Incentive - Practice Incentives Program (PIP) could be made without altering the NBCSP design.

\section{Introduction}

Early cancer detection is a fundamental element of preventive care in Australian primary care. ${ }^{1} \mathrm{~A}$ recommendation from an individual's usual general practitioner (GP) is the most influential factor in a patient's participation in colorectal cancer (CRC) screening. ${ }^{2}$ However, GP involvement in the design of the National Bowel Cancer Screening Program (NBCSP) has been limited. ${ }^{3}$

A body of evidence describes effective interventions aimed at enhancing participation in CRC screening via primary care. ${ }^{1}$ However, to date, little progress has been made in achieving sustained improvements in CRC screening participation in practice. ${ }^{4}$ Little is known about contextual factors that influence whether GPs advise their eligible patients to be screened..$^{5}$ Indeed, in Australia, there has been little empirical research exploring primary care facilitators of, and barriers to, screening since the NBCSP was introduced in $2006^{6}$, reflecting the limited role of primary care in the original program design. ${ }^{3}$

The NBCSP is well established in delivering CRC screening kits (faecal occult blood tests [FOBTs]) to the households of eligible patients (aged 50-74 years) and contributes to reducing CRC morbidity and mortality. ${ }^{7}$ However, the screening participation rate remains one of the lowest in the world at $42 \%$, even though the NBCSP was introduced more than 15 years ago. ${ }^{7}$ Increasing the screening participation rate to at least $60 \%$ (and the rate of diagnostic assessment follow-up to $90 \%$ ) will maximise the mortality benefit from the NBCSP. ${ }^{8}$ Communitytargeted initiatives (such as mass media campaigns ${ }^{9}$ ) have shown some success in increasing screening participation, and primary care is also well placed to reach non-adherent patients for both screening and follow-up.

Our recent systematic review found that interventions that were part of a quality improvement (QI) process maximised use of GPs' influence in recommending CRC screening participation, particularly for underscreened or never-screened patients. ${ }^{6}$ However, the variation in reporting means that it is unclear which $\mathrm{Q}$ l elements contribute to the overall study outcomes. ${ }^{6}$ Based on our review and a general practice survey ${ }^{10}$, we developed a multicomponent CRC-QI intervention comprising eight elements to support delivery of the NBCSP in general practice (Table 1).

Knowing that implementation of a new intervention often fails, we wanted to add flexible implementation strategies into the final design of the CRC-QI intervention (START-CRC). ${ }^{11,12}$ We report on the use of a theory-based tool to explore contextual influencers that might facilitate

Table 1. Elements of the colorectal cancer screening quality improvement (CRC-QI) intervention that support delivery of the NBCSP in general practice

\begin{tabular}{ll} 
Elements of the CRC-QI intervention (START-CRC) & Description \\
\hline Screen (and follow up) eligible, non-adherent patients & $\begin{array}{l}\text { Identify and remind eligible, asymptomatic patients about bowel } \\
\text { cancer screening, with a focus on those who are not up to date }\end{array}$
\end{tabular}

Target a patient cohort and set goals

Audit and feedback

Reorient finance and structure

Training

Collaborate within and across practices

Realign practice roles

Clinical software adjustments
Set goals and targets for bowel cancer screening QI activity

Measure, monitor and review bowel cancer screening clinical data against established practice-specific targets

Use informal and formal reward systems to increase implementation of elements of the CRC-QI intervention

Identify and organise training for a consistent QI and clinical practice approach across all medical and non-medical practice staff

Adopt an internal team and external network approach to share best practice for improvement in bowel cancer screening

Align administrative and clinical team interests to the practice priority of improving bowel cancer screening participation rates

Use clinical systems appropriately to effectively identify and engage patients, extract data reliably and monitor success 
implementation of the intervention. To accommodate existing organisational policies, systems and resources in different practice settings, this study aimed to discover and explore potential challenges to the uptake of the proposed CRC-QI intervention into routine clinical practice.

\section{Methods}

We undertook a qualitative study to explore contextual facilitators of, and barriers to, the implementation of the proposed CRC-QI intervention. The theory-based Consolidated Framework for Implementation Research $(\mathrm{CFIR})^{13}$ guided the formative evaluation.

\section{Participants}

A purposive sampling technique consisting of advertisements via the Adelaide Primary Health Network was used to recruit practitioners from general practices which expressed an interest in improving CRC screening. The study coordinator explained the study verbally to interested respondents, who nominated their general practice to participate.

\section{Setting}

We undertook three focus groups with general practice staff: GPs, practice nurses and administrative staff (e.g. practice managers). Participants were from two general practices in metropolitan Adelaide and one general practice in rural South Australia. Practice staff members were given an explanatory statement and gave written consent before their participation in focus groups. Participants received a $\$ 150$ honorarium to cover the costs associated with their participation.

\section{Focus group discussion}

An independent facilitator conducted a 60-75-minute face-to-face focus group in each general practice at a mutually convenient time in November and December 2018. Focus groups were preferred to other techniques (such as individual interviews), as a more timely and cost-effective approach to collect data from stakeholders (medical and nonmedical practice staff). Furthermore, the group interaction and discussion gave a broader practice perspective on existing CRC screening practices.

The CFIR tool was used to explore diverse contextual influencers and inform the final design of the proposed CRC-QI intervention (START-CRC). The CFIR describes 39 standardised implementation-related constructs, (e.g. relative priority, compatability, time for change, structural characteristics) across five major domains, that interact to influence implementation success in health service settings. ${ }^{13}$ The five domains are intervention characteristics, inner-setting characteristics (characteristics of the implementing organisation), outersetting characteristics (external context), characteristics of individuals, and the implementation process. The CFIR model guided the development of a semistructured interview schedule, incorporating questions identified from our scoping review ${ }^{6}$ and survey. ${ }^{10}$ The interview questions addressed the key CFIR constructs relevant to CRC screening practice across all five domains of the CFIR (Supplementary File 1, available from: doi. org/10.6084/m9.figshare.13395329.v1).

\section{Thematic analysis}

One investigator and the independent facilitator undertook a deductive thematic analysis ${ }^{14}$ of transcriptions and field notes to derive findings in the context of the CFIR model. A six-step process was followed: 1) reading each transcript to become familiar with the data; 2) generating initial codes; 3) identifying themes from the initial codes; 4) reviewing the themes; 5) defining and naming themes; and 6) producing a summary report. ${ }^{15}$ Codes were assigned to participant responses in view of the CFIR constructs. These codes were then organised into themes aligning with the CFIR constructs. A second investigator then checked the codes and themes, and their assignment to the CFIR model. Consensus-based discussion resolved any discrepancies between investigators.

Subsequently, implementation strategies and adaptations to the CRC-QI intervention were identified from the themes. These were discussed with an overseeing Research Advisory Group to ensure transferability and alignment with the local primary care setting. This overall process resulted in changes to the design of the CRC-QI intervention and identified factors that might facilitate implementation across different practice settings. Key findings are highlighted for each theme using exemplar quotes from focus group participants.

\section{Ethics approval}

The study was undertaken as part of a larger study approved by the University of South Australia Human Research Ethics Committee (Application ID: 201061).

\section{Results}

Eighteen GPs and practice staff, from three selfnominated general practices, participated in the focus group discussions (Table 2). All participating practices had more than four GPs. Participants described a range of facilitators of, and barriers to, implementation of the proposed CRC-QI intervention, aligned with the CFIR tool (Supplementary File 2, available from: doi.org/10.6084/ m9.figshare.13395329.v1). Various implementation strategies were also identified to respond to the identified facilitators and barriers (Supplementary File 2, available from doi.org/10.6084/m9.figshare.13395329.v1). Overall, the proposed CRC-QI intervention was positively 
received. Participants noted that many of the CRC-QI elements were already in routine practice. Participants also identified that these elements could be more broadly applied in other preventive health areas, such as cervical screening.

Table 2. Participant characteristics

\begin{tabular}{l} 
Characteristics \\
\hline Gender \\
Male \\
Female \\
Role in general practice
\end{tabular}

GP 12

Nursing

Administration 1

\section{Location}

Urban

Regional

\section{Age}

$<50$ years

$\geq 50$ years

6

Across all focus group discussions, the following five themes were consistently identified that have the potential to influence several elements of the proposed CRC-QI intervention.

\section{Priority setting and incentives}

Participants reported that existing general practice programs and activities that were incentivised via payments or requirements for practice accreditationbased incentives would make implementing the proposed CRC-QI intervention a low priority. For example, activities such as allergy recording, cycles of care (e.g. for diabetes), patient demographic (e.g. student population and travel medicine) and activities related to specific clinical interests (e.g. women's health) would be prioritised over CRC screening where no payment or incentive exists. Individual practices and individual medical staff reported differing priorities or goals:

... it's part of our senior training....we're supposed to do an audit, every 3 years, and that's why I do mine on [clinical interest] audits ... (urban, GP)

... it's a requirement as well for the practice as part of our accreditation. We undertake an [clinical interest] audit and improvement activity every 3 years as well. (urban, administration)

Furthermore, participants noted that staff with specific interests tend to informally become the 'go-to' people for those specific clinical areas (e.g. immunisation), reducing the perceived need for others in the practice to be active in that area:

... I think if there's somebody who is sort of the focus of it, that is their kind of thing that they're looking after particularly, then it helps keep us on track. (regional, GP)

However, despite such embedded priority setting, no defined targets were described:

We don't actually have any sort of strict goals or targets exactly but we certainly have a focus on what we want to look at. (urban, GP)

Some participants also noted that several nongovernment efforts and groups were working towards increasing CRC screening awareness. These activities could deter GPs from adding to these efforts and making CRC screening a priority for their practice:

There's a couple of community groups who run FOBTs occasionally too so then we have four or five different groups trying to get people in for their FOBTs. If they [patient] don't respond to your recall because they've already done it with the [community group], and we don't get the results of that. (regional, GP)

Interestingly, when participants were made aware from the study recruitment information of the suboptimal CRC screening participation rate in Australia (42\%), they noted this as a motivator for change and a reason to prioritise CRC screening. Participants suggested attaching a financial or accreditation incentive to also motivate nonmedical and administration staff to prioritise CRC screening and improve the likelihood of practice uptake:

Admin staff and nursing staff, they get motivated to become a part of the process because there's that payment associated with it which perhaps could be one thing that when it comes to bowel cancer and breast cancer screening cycle of care. (regional, GP)

The findings suggest that identifying nursing or administration staff with an interest in CRC or screening may help to coordinate the START-CRC implementation process. Such key coordination roles would support medical staff in adopting the intervention and facilitate effective implementation.

\section{Information technology}

Participants reported that electronic clinical record systems increase the safety, quality and efficiency of care overall. However, these systems currently lack features and facilities to effectively identify and engage eligible, 
non-adherent patients, and enable CRC screening audit and feedback mechanisms:

To work out whether or not somebody's screening is up to date, that would be cumbersome.

(regional, GP)

Well, there is ... a screening part of [audit tool] but I'm not sure if it does have bowel screening, but I can't work out where you'd put it in [clinical software], and it's possible that there isn't somewhere for us to be able to get that data. (urban, administration)

Deficiencies in clinical software were reported as significant barriers across both inner settings (individual and practice) and outer settings (system levels), and several elements of the proposed CRC-QI intervention. These deficiencies could potentially dictate how much organisational change could be implemented. Barriers include poor enforcement of data entry consistency, variable data extraction capability, and limitations in system configurations for prompts and data entry for CRC screening

[The CRC screening result] comes in and you discuss it with the patient. But, I don't know if there's a tool to actually extract it. (urban, GP)

Some IT support was available from both practice support networks (such as the Primary Health Networks) and software providers. However, practices with fewer resources were often less able to access this support. Furthermore, the software providers were reported to only act on requests for changes to clinical systems when many user requests were received.

\section{Patient-level barriers}

Participants consistently commented that patient education to make CRC screening a priority, among other personal health responsibilities, appears to be low:

And they get a pack home, you ask them have they done it. They go: "no, because it's just a pack to tell you, you know, you need to do this and this, and there's no other explanation, no other reason, no stats, nothing at all, to guide that it's a priority". (urban, nurse)

Additional barriers include appointment time and purpose, from both the patient and professional perspective, and the confronting nature of the at-home FOBT:

It's actually patients not wanting to deal with 'the poo thing' so it's talking through how to collect it and some of them are like: "I'm just not going to do that". (regional, GP)

Participants agreed that effectively engaging patients was fundamental to the effectiveness of the proposed CRC-QI intervention:
Seems like it is about education because people accept that you have to do cervical screening, you have to do breast screening. (regional, GP)

Routinely incorporating educational material and opportunistically instigating CRC screening conversations were suggested to help patients prioritise CRC screening and encourage them to routinely participate in the NBCSP, if appropriate. GP participants acknowledged their ability to influence patients to participate in cancer screening but noted that inadequacies of their clinical information systems, combined with crowded agendas in appointments, often meant that they did not advise patients to undertake screening. Similarly, participants commented on the problem of sometimes not knowing why a patient failed to respond to practice outreach - for example, when they had already undergone screening elsewhere:

There's a couple of community groups who run FOBTs occasionally too so then we have four or five different groups trying to get people in for their FOBTs. If they don't respond to your recall because they've already done it with the [community group], and we don't get the results of that. (regional, GP)

Automated recalls and prompts would help to increase screening recommendations and divert some responsibility from GPs to practice nurses and nonmedical staff. However, clarity about who is responsible for follow-up of patients participating in the NBCSP was needed. While the NBCSP takes on some of this follow-up role, best practice care would require that the treating doctor normally determines the follow-up depending on the severity of the risk.

\section{Clinical practice}

Participants agreed that clinical data were generally not used to understand suboptimal practice, unless it was a priority area. Current practice-level CRC participation rates and the number of eligible, non-adherent patients were unknown among participants; this information would be a determining factor in implementing the proposed CRC-QI intervention. Despite agreement that CRC screening should be a practice-level priority, practitioner variation is likely to exist in CRC screening practice. Participants noted that individual, competing priorities and time constraints would lead to differences in policy and system adherence:

... there'd still be variations in the way the doctors would document reminders and that sort of thing, and use of the action box. I know that not every doctor uses the action box. So there's certainly variation there. (urban, GP)

Furthermore, there was some confusion among participants regarding CRC screening intervals, possibily arising from the differing NBCSP implementation phases. 
GP participants also expressed concern about a potential increase in wait times for diagnostic (colonoscopy) assessments as a perceived unintended consequence of increasing CRC screening participation following the implementation of the proposed CRCQI intervention. Participants indicated a reluctance to increase screening efforts until waiting times for colonoscopies decrease:

I think one of the problems we have ... we do have a lot of people with positive testing and getting access to colonoscopies is very, very tough. (urban, GP)

Most patients prefer public because of cost. And, the public waiting list for gastroenterology is really, really long. (urban, nurse)

Furthermore, the lack of robust data, from both the practice (number of non-adherent patients) and the hospital (publicly available waiting times) made it difficult to quantify the effect of increasing screening recommendations on colonoscopy waiting times and advise patients about the colonoscopy waiting time after receiving a positive FOBT. Having GPs work more closely with gastroenterologists and hospitals to clarify colonoscopy waiting times was proposed to better manage both patient and practitioner expectations.

\section{National Bowel Cancer Screening Program}

Among the participants, there was variable confidence in, and confusion about, the nationally run $\mathrm{CRC}$ screening program (the NBCSP). The inconsistency of the NBCSP implementation intervals with clinical guidelines over the past few years (for example, the current recommended strategy for population CRC screening is an FOBT every two years in asymptomatic individuals aged 50 to 74 years, yet the phased NBCSP implementation meant that initially not all patients were invited) have created some uncertainty about screening intervals, compounded by poor access to patients' CRC screening histories:

... the difference in understanding of the current Red Book [the Royal Australian College of General Practitioners guidelines for prevention activities in general practice] requirements around some of the screenings in the team ... [need] to make sure we're all consistent and up to where the current clinical standard is. (urban, GP)

Furthermore, there was a perceived lack of communication about NBCSP processes, including screening participation rates, to directly support preventive care in primary care. Many participants noted that some implementation barriers could be partly alleviated if the NBCSP and National Cancer Screening Register had greater functionality and completeness:

That depends on us being able to get on and see the registry so we're not sending out wasteful letters. (regional, GP)
Several fundamental QI practices were suggested that are not currently available through the screening register. These include capturing patients participating in CRC screening beyond the NBCSP, identifying ineligible patients through Medicare Benefits Schedule data linkage (e.g. total colectomy or surveillance programs) and communicating participation rates with primary care. Integrating existing clinical/practice management software systems to give GPs access to screening histories, and identifying and reminding non-adherent patients in a similar way to what is done in other preventive health areas (such as cervical screening and immunisation) were also suggested:

If we can log on [to the screening register] like we do with the immunisation register, that would be fantastic. (regional, GP)

\section{Discussion}

The CFIR allowed an evaluation of general practice perspectives to inform adaptations of the proposed CRCQI intervention before implementation. Practices were already implementing elements of the CRC-QI intervention as part of their usual practice, but the CFIR identified where practices would require tailored implementation strategies. The findings suggest that primary care can support the NBCSP by adaptating existing systems, when barriers and facilitators are appropriately recognised. The CFIR provides a practical framework to capture the many facilitators and barriers, ranging from technological issues to patient-level barriers, and also identifies several key themes relevant across practice settings. These broader determinants include both inner-setting influencers (practice priorities and incentives; information technology) and outer-setting contextual influencers (such as the NBCSP).

The CFIR-guided discussion highlights the range of interconnected barriers to implementation of interventions in primary care settings ${ }^{16,17}$ that may need to be overcome to improve general practice involvement in CRC screening. ${ }^{4}$ The unique perspective offered by the CFIR highlights both the challenges associated with implementing interventions in primary care ${ }^{11}$ and the potential for more GP involvement in the design of the NBCSP. ${ }^{3}$

Importantly, the proposed CRC-QI intervention was well received, with many elements already implemented by general practices for other aspects of preventive care, for example automated recalls for cervical screening. The implementation strategies identified were aligned to stakeholder interests, which are an important facilitator of implementation ${ }^{12}$, and which vary according to existing practices and resourcing. A key intervention characteristic, which crossed several CFIR domains, was the need for clinical software adjustments (the final ' $C$ ' of START-CRC). Systems, training and ongoing support in this QI element are not unique to START-CRC, although 
the study highlights the individual and practice variation in this routine clinical practice element. An indirect benefit of our study is the general practice 'buy-in', potentially enabling relevant and transferrable 'co-created' outcomes and sustainable partnerships that are imperative for implementing effective change within primary care. ${ }^{18}$

An acknowledged study limitation is a potential participant selection bias, which may limit the transferability of our findings and proposed modifications to the CRC-QI intervention. Practices were recruited from respondents with a self-identified interest in bowel cancer screening, although the nature of the bias is difficult to define. Furthermore, no practices with a single GP were involved because the limited nursing or administrative support precluded study participation, and may also impose barriers to implementation of the CRC-QI intervention. ${ }^{10}$

The CFIR-guided discussion highlighted operational variability across even a few general practices, emphasising the need for a flexible implementation approach to the screening intervention. We involved all stakeholder groups, whereas many studies focus only on the medical staff. This broader practice perspective allowed nursing and administrative staff to confirm processes that GPs may not always be clear about.

Interventions that incorporate multicomponent or Q। practices tend to be more effective, particularly if they move the responsibility for change from clinical staff to other parts of the system. ${ }^{19,20}$ Our study also highlights that an inherent commitment to organisational change - at both the individual and practice level - is needed to address some intangible elements, such as priority setting. ${ }^{21,22}$ Our findings build on our previous work showing that the existing resource level to implement QI activity in part shapes CRC screening recommendations in general practice. ${ }^{10}$ It is hypothesised that the proposed CRC-QI intervention will improve CRC screening participation in practices with sufficient existing resources to implement a multifaceted QI approach targeting CRC screening; this warrants further investigation.

Although primary care involvement in the original NBCSP design was limited, there has been more recent effort to engage the primary care workforce, with the release of an NBCSP Primary Care Engagement Strategy in 2016. ${ }^{23}$ However, the practical support offered tends to focus on changes within general practice to maintain the fundamental NBCSP design. Importantly, outersetting constructs beyond the practice setting (such as changes to external policy and incentives) may better engage the primary care workforce in the NBCSP. For example, the new National Cancer Screening Register ${ }^{24}$ might allow GPs to readily access screening histories (particularly for new patients) or capture those patients participating in CRC screening beyond the NBCSP. Similarly, if the Australian Government's general practice Quality Improvement Incentive - Practice Incentives Program (PIP) 25 incorporated CRC screening, practices may focus organisational activity on identifying non- adherent patients and improving screening participation rates within their patient population. Modification of both established government initiatives may allow better integration of current clinical practice with NBCSP activity without needing to alter the program design.

The next phase of our study aims to validate these findings in a larger-scale implementation study to evaluate the effectiveness and sustainability of the CRC-QI intervention. The ability to implement policy change through screening registries and $\mathrm{Q}$ incentive programs that work alongside the NBCSP will encourage a coordinated approach, and offset the challenges of implementing change directly within the primary care setting. ${ }^{12}$ Future research needs to focus on QI practices targeting CRC screening and move the responsibility for change to all parts of the system, both internal and external to general practice.

\section{Conclusions}

GPs could play an important adjunct role to the NBCSP, accommodated though adaptation of existing Q। programs rather than redesign of the organised CRC screening program per se. Our study offers a unique contextual perspective to inform strategies aimed at promoting $\mathrm{CRC}$ screening in general practice to achieve more active engagement of the primary care workforce, working alongside the NBCSP.

\section{Acknowledgements}

We thank the GPs and their practice staff who generously gave their time and insight to this study. We also acknowledge Cancer Council SA's Beat Cancer Project for financial support of this project, and the support and assistance of Mint Research Pty Ltd in the facilitation and preliminary analysis of the focus group discussions. The funding body had no input into the study design or reporting of study findings.

The input of other members of the Research Advisory Group, Professor Richard Reed, Professor Caroline Miller and Professor David Roder, is also acknowledged. This project was undertaken as part of the No Australians Dying of Bowel Cancer Initiative (NADBCI). The NADBCI wishes to acknowledge the Medical Research Future Fund and the Australian Government Department of Health for financial support for the broader project aim to eradicate bowel cancer death in Australia.

\section{Peer review and provenance}

Externally peer reviewed, not commissioned.

\section{Competing interests}

None declared. 


\section{Author contributions}

$\mathrm{CH}$ was involved in the conception and design of the study, gaining funding and ethics approval. $\mathrm{CH}$ coordinated the project, undertook initial thematic analysis and drafted the manuscript. OF provided insights to the analysis and helped draft the manuscript. DT oversaw thematic analysis and interpretation, and helped draft the manuscript. 10 was involved in the study conception and design, gaining funding and ethics approval. IO oversaw the project and helped draft the manuscript. All authors reviewed and approved the final manuscript.

\section{References}

1. Emery JD, Shaw K, Williams B, Mazza D, FallonFerguson J, Varlow M, Trevena LJ. The role of primary care in early detection and follow-up of cancer. Nat Rev Clin Oncol. 2014;11:38-48.

2. Zajac IT, Whibley AH, Cole SR, Byrne D, Guy J, Morcom J, Young GP. Endorsement by the primary care practitioner consistently improves participation in screening for colorectal cancer: a longitudinal analysis. J Med Screen. 2010;17:19-24.

3. Grogan PB, Olver IN. A bowel cancer screening plan at last. Med J Aust. 2014;201:435-6.

4. Dodd N, Mansfield E, Carey M, Oldmeadow C, SansonFisher R. Have we increased our efforts to identify strategies which encourage colorectal cancer screening in primary care patients? A review of research outputs over time. Prev Med Rep. 2018;11:100-4.

5. Davis MM, Freeman M, Shannon J, Coronado GD, Stange KC, Guise J-M, et al. A systematic review of clinic and community intervention to increase fecal testing for colorectal cancer in rural and low-income populations in the United States - how, what and when? BMC Cancer. 2018;18:40.

6. Holden CA, Frank O, Caruso J, Turnbull D, Reed RL, Miller CL, Olver I. From participation to diagnostic assessment: a systematic scoping review of the role of primary health care in the National Bowel Cancer Screening Program. Aust J Prim Health. 2020;26:191206.

7. Australian Institute of Health and Welfare. National Bowel Cancer Screening Program: monitoring report 2020. Canberra: AlHW; 2020 [cited 2020 Dec 8]. Available from: www.aihw.gov.au/reports/cancer-screening/nationalbowel-cancer-screening-monitoring-2020/contents/tableof-contents

8. Worthington J, Lew J-B, Feletto E, Holden CA, Worthley DL, Miller C, Canfell K. Improving Australian National Bowel Cancer Screening Program outcomes through increased participation and cost-effective investment. PLoS ONE. 2020;15:e0227899.
9. Durkin S, Broun K, Guerin N, Morley B, Wakefield M. Impact of a mass media campaign on participation in the Australian bowel cancer screening program. J Med Screen. 2020;27:18-24.

10. Holden CA, Frank O, Li M, Manocha R, Caruso J, Turnbull $D$, et al. Engagement of general practice in an Australian organised bowel cancer screening program: a cross-sectional survey of knowledge and practice. Asian Pac J Cancer Prev. 2020;21:2099-107.

11. Craig P, Dieppe P, Macintyre S, Michie S, Nazareth I, Petticrew M. Developing and evaluating complex interventions: the new Medical Research Council guidance. Br Med J. 2008; 337:a1655

12. Lau R, Stevenson F, Ong BN, Dziedzic K, Treweek S, Eldridge $S$, et al. Achieving change in primary carecauses of the evidence to practice gap: systematic reviews of reviews. Implement Sci. 2016;11:40.

13. Damschroder LJ, Aron DC, Keith RE, Kirsh SR, Alexander JA, Lowery JC. Fostering implementation of health services research findings into practice: a consolidated framework for advancing implementation science. Implement Sci. 2009;4:50.

14. Patton MQ. Qualitative research and evaluation methods. Thousand Oaks, CA: Sage Publications; 2002.

15. Braun V, Clarke V. Using thematic analysis in psychology. Qual Res Psych. 2006;3:77-101.

16. The Community Preventive Services Task Force. Cancer screening: multicomponent interventions - colorectal cancer. US: CPSTF; 2016 [cited 2019 July 15]. Available from: www.thecommunityguide.org/findings/cancerscreening-multicomponent-interventions-colorectalcancer

17. Harris DM, Borsky AE, Stello B, Johnson M, Gratz N, Sarfaty $M$, et al. Tracking and improving screening for colorectal cancer intervention: a systems approach. Rockville, MD: Agency for Healthcare Research and Quality; 2012 [cited 2019 July 15]. Available from: www. ahrq.gov/sites/default/files/wysiwyg/research/findings/ final-reports/crctoolkit/crctoolkit.pdf

18. Janamian T, Crossland L, Jackson CL. Embracing value co-creation in primary care services research: a framework for success. Med J Aust. 2016;204:S5.

19. Klabunde CN, Lanier D, Breslau ES, Zapka JG, Fletcher RH, Ransohoff DF, Winawer SJ. Improving colorectal cancer screening in primary care practice: innovative strategies and future directions. J Gen Intern Med. 2007;22:1195-205.

20. Senore C, Inadomi J, Segnan N, Bellisario C, Hassan C. Optimising colorectal cancer screening acceptance: a review. Gut. 2015;64:1158-77.

21. Weiner BJ. A theory of organizational readiness for change. Implement Sci. 2009;4:67. 
22. Arroyave AM, Penaranda EK, Lewis CL. Organizational change: a way to increase colon, breast and cervical cancer screening in primary care practices. J Community Health. 2011;36:281-8.

23. Australian Government Department of Health. National Bowel Cancer Screening Program - Primary Health Care Engagement Strategy, 2016-2020. Canberra: DoH; 2015 Oct [cited 2020 Dec 8]. Available from: www.health. gov.au/resources/publications/national-bowel-cancerscreening-program-primary-health-care-engagementstrategy
24. Australian Government Department of Health. National Cancer Screening Register. Canberra: Australian Government; 2019 [cited 2019 Sept 3]. Available from: www.ncsr.gov.au

25. Australian Government, Services Australia. Practice Incentives Program. Canberra: Services Australia; 2020 [cited 2020 Dec 21]. Available from: www. servicesaustralia.gov.au/organisations/healthprofessionals/services/medicare/practice-incentivesprogram

\section{Copyright: (C) $(1)(0)$}

(C) 2020 Holden et al. This article is licensed under the Creative Commons Attribution-NonCommercial-ShareAlike 4.0 International Licence, which allows others to redistribute, adapt and share this work non-commercially provided they attribute the work and any adapted version of it is distributed under the same Creative Commons licence terms. See: www.creativecommons.org/licenses/by-nc-sa/4.0/ 